\title{
Article \\ Semi-Experimental Determination of the Linear Clamped Electro-Optical Coefficients of Polar Crystals from Vibrational Spectroscopic Data
}

\author{
Eric Bouhari ${ }^{1,2}$, Ballo Mohamadou ${ }^{2, *,+}$ (D) and Patrice Bourson ${ }^{3}$ (D) \\ 1 Centre de Formation Professionnelle d'Excellence, Sangmelima P.O. Box 858, Cameroon; \\ bouharieric@yahoo.fr \\ 2 Higher Teacher's Training College, The University of Maroua, Maroua P.O. Box 46, Cameroon \\ 3 Laboratoire Matériaux Optique Photonique et Systèmes, LMOPS-EA 4423, Université de Lorraine et \\ CentaleSupélec, 2 Rue E. Belin, 57070 Metz, France; patrice.bourson@univ-lorraine.fr \\ * Correspondence: ballomohamadou@aol.fr \\ + Principal Investigator.
}

check for updates

Citation: Bouhari, E.; Mohamadou, B.; Bourson, P. Semi-Experimental Determination of the Linear Clamped Electro-Optical Coefficients of Polar Crystals from Vibrational Spectroscopic Data. Crystals 2022, 12, 52. https://doi.org/10.3390/ cryst12010052

Academic Editor: Shujun Zhang

Received: 13 November 2021 Accepted: 16 December 2021 Published: 31 December 2021

Publisher's Note: MDPI stays neutral with regard to jurisdictional claims in published maps and institutional affiliations.

Copyright: (c) 2021 by the authors. Licensee MDPI, Basel, Switzerland. This article is an open access article distributed under the terms and conditions of the Creative Commons Attribution (CC BY) license (https:// creativecommons.org/licenses/by/ $4.0 /)$.

\begin{abstract}
The present work highlights a new general method devoted to computations of the clamped linear electro-optical coefficients from the measured fundamental vibrational frequencies and the nonlinear dielectric susceptibility constants. The calculations are based on the formula analog to that of the Lyddane-Sachs-Teller relation, which is systematically used for the calculations of the clamped linear electro-optical coefficient of oxide ferroelectric crystals such as $\mathrm{LiNbO}_{3}, \mathrm{LiTaO}_{3}$, $\mathrm{BaTiO}_{3}, \mathrm{PbTiO}_{3}$, and $\mathrm{KNbO}_{3}$. The computed electro-optical coefficients are in good agreement with those obtained from direct measurements and the first-principles calculations or other semi-empirical models. In addition, the famous $r 51$ or $r 42$ coefficients of the tetragonal $\mathrm{BaTiO}_{3}, \mathrm{PbTiO}_{3}$, and $\mathrm{KNbO}_{3}$ crystals are finally calculated with high accuracy and discussed in connection with the soft mode behavior.
\end{abstract}

Keywords: electro-optics; vibrational mode; ferroelectric materials

PACS: 78.20.Jq 63.70.+h 42.70.Mp

\section{Introduction}

The linear electro-optic (Pockels) effect is the change of the refractive indices under the applied electric field of low frequency through its coupling with an exciting electric field. Generally, the magnitude of the effect varies with the polarization of both the electric field and the optical wave, depending on the crystal symmetry of the electro-optic (EO) material. This concept was first invoked by Poulet [1] and later by Loudon [2] to explain the difference between the longitudinal and transverse phonon powers at the zone center. Because of its importance in light field modulation [3], it results in a broad range of technological applications [4]: for example, photonic quantum devices that monitor and control photon quantum states $[5,6]$. The linear EO effect is materialized by coefficients that are components of a $6 \times 3$ tensor in its reduced form. The EO coefficients of lithium niobate, $\mathrm{LiNbO}_{3}(\mathrm{LN})$, and other ferroelectric crystals will be the objective of the present study. These EO coefficients can be directly measured for crystals of satisfactory sizes and optical quality. However, they can be indirectly and accurately determined from Raman and infrared (IR) reflection spectroscopic data [7-9]. EO coefficients and nonlinear optical (NLO) coefficients are also calculated by density functional theory (DFT) in the framework of the first-principles study $[10,11]$, which turn out to be self-consistent. The NLO coefficients can be semi-empirically calculated using the bond charge model (BCM) [12]. Based on this model, Shu and Yariv [13] derived some improvements which are currently used for the electro-optical calculation of a large band of NLO crystalline materials [14,15]. Another 
equivalent model, the bond orbital model (BOM) [16], could also, in principle, be modified to determine the EO coefficients in semiconductors and insulating materials. There are other semi-empirical models [17-19] devoted to the calculations of both EO and NLO coefficients. However, we will only focus our comparison on the output of the calculation based on the ab initio DFT and on BCM that requires, as a starting point of the computations, only the crystallographic structural data such as the atomic positions or the crystal bond lengths. Kaminow [20] first pointed out a relationship that conveys the calculations of the EO coefficients from the measured nonlinear dielectric susceptibility coefficients (d-NLO), the Raman polarizabilities, and the effective charge parameter. Although this relation provides EO coefficients that are very close to those obtained from direct measurements, there is one important difficulty: the correct algebraic value of the Raman polarizability. The absolute value of a given Raman tensor component is extracted from the stringent measurements of the Raman intensity up to a sign. The problem of sign indetermination is solved by establishing sign decision criteria mainly based on statistical considerations $[8,9]$. In this work, we will show that there is no need to resort to these experimental protocols often associated with unavoidable errors. These experimental procedures work very well for the calculation of the EO coefficient of a wide class of binary compounds that exhibit one to three activated Raman lines [21]. Up to now, no report publishes the correct value of the $r_{51}$ coefficient of barium titanate $\mathrm{BaTiO}_{3}$ and lead titanate $\mathrm{PbTiO}_{3}$ perovskite crystals.

In this work, we establish a new Lyddane-Sachs-Teller relation, namely the nonlinear LST relation, and then we test this relation by calculating the EO coefficients of the oxide ferroelectric of interest and, in particular, the $r_{51}$ coefficients of the tetragonal $\mathrm{BaTiO}_{3}$, $\mathrm{PbTiO}_{3}$, and potassium niobate $\left(\mathrm{KNbO}_{3}\right)$ oxide ferroelectrics, and we give a sound interpretation of the exceptional high values of these coefficients. This work can be considered as a semi-experimental method of the determination of EO coefficients since it requires, before the calculations, the measurements of the vibrational frequencies, e.g., the longitudinal optic (LO) and transverse optic (TO) frequencies and their corresponding damping coefficients (or linewidths). The theoretical part of this work is sub-tended by the complex nonlinear dielectric response function (CNLDF), which is formerly established on the base of the dynamical crystal lattice considerations. By taking advantage of the product form of this CNLDF, we derived another version of the LST relation, which is systematically used for the computations of EO coefficients, and finally we show that the relative errors of the indirectly measured EO coefficients could only depend on the measured Raman peak linewidth and its associated wavenumber.

This report is arranged as follows. Section 2 is devoted to the crystal optics formulation of the complex nonlinear dielectric function, allowing for the derivation of the final expressions of the EO coefficients, the Miller indices, and the relative errors. Section 3 is dedicated to results, and the discussion is divided into two parts. The first part presents and discusses the results concerning $\mathrm{LiNbO}_{3}(\mathrm{LN})$ and $\mathrm{LiTaO}_{3}$ (LT) crystals, while the second part relies on the calculations of the $\mathrm{EO}$ coefficients of some oxide perovskite crystals such as $\mathrm{BaTiO}_{3}(\mathrm{BTO}), \mathrm{PbTiO}_{3}(\mathrm{PTO})$, and $\mathrm{KNbO}_{3}(\mathrm{KNO})$. In addition, the $r_{42}$ or $r_{51}$ coefficients of the BTO and PTO crystals, never accurately calculated up to now, are given and their extremely high values are explained in the light of the low-frequency spectral part.

\section{Method}

\subsection{The Complex Nonlinear Dielectric Response Function (CNLDF)}

In a principal coordinate system with optical dielectric tensor $n_{i j}^{2}=n_{i}^{2} \delta_{i j}$, the Pockels electro-optic effect is defined without the sum convention [21]

$$
\Delta\left(n^{-2}\right)_{i j}=\sum_{k} r_{i j k} E_{k}(\omega)
$$

where $E_{k}(\omega)$ is the modulating electric field at the angular frequency $\omega$, and $r_{i j k}$ is the Pockels EO coefficient which is a constant independent of the modulating electric field frequency. The modulating electric field can be created by an electric potential applied 
to the crystal. In the absence of an applied electric field, only the macroscopic electric field inside the crystal will induce the associated polarization. We will consider only the latter case for the purpose of the present study. The $r_{i j k}$ constant can be experimentally or computationally determined. The main goal of this section relies on how to determine the accurate value of $r_{i j k}$ coefficient.

To this end, let us consider a crystal structure with $\mathrm{N}$ number of atoms per unit cell. The density-weighted Cartesian coordinates of the crystal atom's positions X can be transformed into normal coordinates through an algebraic relation [22]. Under these assumptions, the equation of the damped motions inside the crystal subjected to a harmonic potential is

$$
\vec{W}+\Gamma \vec{W}+\Omega \vec{W}=Z \vec{E}
$$

where $\Omega$ is the diagonal matrix of the TO squared frequencies, $\vec{E}$ is the macroscopic electric field vector, $\vec{W}$ is the normal coordinates vector, $Z$ is a $3 N \times 3$ matrix of the transverse effective charge parameter which can be constructed from $3 \times 3$ submatrices of the Born effective charges in the unit cell, and $\Gamma$ is the diagonal matrix of the mode damping coefficients.

According to $[7,9]$, the macroscopic susceptibility in most polar crystals depends on both the normal coordinates and the macroscopic electric field. As a consequence, the Raman scattered intensity will have two contributions: the most dominant contribution is due to the change of the linear crystal susceptibility by the normal coordinates, and an additional contribution arises from the macroscopic electric field which induces an associated polarization. Depending on the frequency of the modulating electric field, it can result in optical rectification, optical second harmonic generation (SHG), and three-wave interaction $[9,21]$. From an alternate point of view, the transition susceptibility $\left[\chi_{\alpha \beta}\right]_{S}$ from the initial state $\left(\omega_{l}\right)$ to the final state $\left(\omega_{s}\right)$ causes a polarization with mixed frequency [9]. In this context, one can imagine the Raman scattering by a nonlinear optical crystal as an analog of two types of three waves mixing, that is, the laser electric field $E_{l}\left(\omega_{l}\right)$ and the two scattered electric fields $E\left(\omega_{s}\right)$, with $\omega_{s}=\omega_{l}+\omega$ for the anti-Stokes Raman scattering process and $\omega_{s}=\omega_{l}-\omega$ for the Stokes Raman scattering process, $\omega$ being the ionic vibrational frequencies that are situated well below the electronic resonance frequencies but sufficiently high enough to avoid piezoelectric resonances. Because of this, the threewave interactions can ultimately be imaged as a spontaneous parametric up-conversion and down-conversion. Based on this representation, the momenta conservation is also satisfied since, in the limit of infinite wavelengths, the modulus of the phonon wave vector can be neglected compared to that associated with the incident and scattered waves: thus, both type I and type II phase-matching conditions are satisfied. Under these situations, the total induced polarization can be therefore written as

$$
\vec{P}=\vec{P}^{L}+\frac{1}{2} \vec{P}^{N L}=\epsilon_{0} \chi \vec{E}+\frac{1}{2} \epsilon_{0} \chi^{N L} \vec{E}_{l} \vec{E}^{*}
$$

where $\vec{P}^{L}$ and $\vec{P}^{N L}$ are the linear and nonlinear induced polarization, respectively, and $\chi$ and $\chi^{N L}$ are the macroscopic linear and nonlinear susceptibility tensors with dimensions 2 and 3 , respectively. Finally $E_{l}$ and $E^{*}$ are, respectively, the laser and complex conjugate of macroscopic electric fields along the principal dielectric axes. The classical Raman scattering is due to the fluctuations of the linear dielectric susceptibility by the crystal normal coordinates, while the nonlinear effect of the Raman scattering is assumed to originate from the fluctuations of the linear susceptibility by the macroscopic interacting electric fields with the nonlinear crystal [9], that is,

$$
\chi^{N L}=\frac{d \chi}{d \vec{E}}=\left[\frac{d \chi}{d \vec{E}_{l}}+\frac{d \chi}{d \vec{E}^{*}}\right]
$$


for the anti-Stokes Raman scattering process with

$$
\frac{d \chi}{d \vec{E}_{l}}=\chi_{N L}^{\infty}=2 d
$$

where $\mathrm{d}$ is the nonlinear optical dielectric susceptibility tensor. Performing the following differentiation rule [23] results in

$$
\frac{d \chi}{d \vec{E}^{*}}=\frac{d \chi}{d \vec{E}_{l}}+\frac{\partial W^{*}}{\partial \vec{E}^{*}} \frac{\partial \chi}{\partial W}
$$

If $\chi$ is a real symmetric tensor, it can be shown that $d E^{*}=d E^{\dagger}=d E$, where $d E^{\dagger}$ is the hermitian conjugate of $d E$. The factor $\partial W^{*} / \partial \vec{E}^{*}$ can be expressed from the vectorial damped harmonic motion Equation (2), and by using this ansatz, (4) is finally written as

$$
\frac{d \chi}{d E}=4 d+Z\left(\Omega-\omega^{2}+i \Gamma \omega\right) a
$$

for the anti-Stokes Raman scattering by optical phonons, and

$$
\frac{d \chi}{d E}=4 d+Z\left(\Omega-\omega^{2}-i \Gamma \omega\right) a
$$

for the Stokes Raman scattering process; this is done by replacing $\omega$ with $-\omega$.

In the above formulas, $a$ is the Raman polarizability tensor defined by $a=\frac{\partial \chi}{\partial W}$.

Despite the apparent difference between (7) and (8), however, these equations lead, as will be seen in the next subsection, to equivalent results. Relation (6) can be written in terms of tensor components as

$$
\chi_{i j k}^{N L}(\omega)=2 \chi_{i j k}^{\infty}+\sum_{m=1} \frac{a_{i j, m} Z_{k, m}}{\omega_{T O, m}^{2}-\omega^{2}+i \gamma_{T O, m} \omega}
$$

with $\chi_{i j k}^{\infty}=2 d_{i j k}$, where $\chi_{i j k}^{N L}$ is the complex nonlinear susceptibility tensor element, $d_{i j k}$ is the high frequency nonlinear dielectric susceptibility tensor component (d-NLO), $Z_{k, m}$ is the effective charge parameter matrix, $a_{i j, m}$ is the mode Raman polarizability tensor component, $\omega_{T O, m}$ is the transverse optical frequency, and $\gamma_{T O, m}$ is the linewidth of the $\mathrm{m}^{\text {th }}$ mode. It is interesting to stress that relation (9) can be extended to the polaritons regions [9]. Since the relative Raman intensity [23] arises from the imaginary part of (9), under the causality principle, one could take advantage of the Kramers-Kronig relations to calculate the real part of relation (9) from the experimental Raman spectrum due to the nonlinear effect.

\subsection{Electro-Optical Coefficient and Relation with the CNLDF}

The change of the inverse of the permittivity tensor due to the total macroscopic electric field is $[11,20]$

$$
\rho=\frac{d}{d E}\left(\frac{1}{\epsilon}\right)=-\frac{1}{\epsilon^{2}} \frac{d \epsilon}{d E}
$$

where $\rho$ is the nonlinear impermittivity tensor.

It can be observed that the EO coefficients are components of a tensor that can be reduced owing to the Voigt rule as an array of $3 \times 6$ elements, contrary to the $\mathrm{d}$ tensor which is given as an array of $6 \times 3$ elements in its reduced form. On the other hand, it has been clearly shown elsewhere [11] that $\epsilon^{2}=\epsilon_{0} \delta_{i l} n_{i}^{2} \delta_{j m} n_{j}^{2}$ with $n_{i, j}$ being the refractive indices along the principal dielectric axes. As $\epsilon=I+\chi$, where $\mathrm{I}$ is the identity matrix, 
the tensor component corresponding to the anti-Stokes nonlinear susceptibility, inserted into (9), finally yields

$$
-n_{i}^{2} n_{j}^{2} \rho_{i j k}(\omega)=4 d_{k i j}+\sum_{m=1} \frac{Z_{k, m} a_{i j, m}}{\omega_{T O, m}^{2}-\omega^{2}+i \gamma_{T O, m} \omega}
$$

If one sets $\omega=0$ in (11), the familiar formula dedicated to the calculation of the EO Pockels coefficient is retrieved with $\rho_{i j k}(0)=r_{i j k}$, where $r_{i j k}$ are the Pockels EO tensor components that need four parameters, among them the square root of the Raman polarizabilities, whose experimental determination is very complicated. Based on Formula (11), the Pockels EO coefficients of some uniaxial crystals are currently calculated using complicated software based on the DFT method $[10,11,24]$, except for the $r_{51}$ Pockels EO coefficient of oxide perovskites.

The imaginary part of $\chi_{i j k}(\omega)$ invokes the Raman intensity due to the nonlinear effect of the Raman scattering by phonon-like polaritons [23], while $\operatorname{Im}\left(\chi_{i j k}(0)\right)$ could be related to the nonlinear analog of the dielectric loss. It may happen that owing to the group theoretical selection rules, some Raman tensor components have a minus sign. Relation (11) is only valid for crystal point groups with associated irreducible representations that are both Raman and IR active. However, if there are exceptionally only IR active modes, as this happens for one of the irreducible representations of the $\mathrm{D}_{3}$ (or 32) point group or for the ungerade modes, $r_{i j k}$ can be calculated by performing the tensor product of the effective charge vectors. In this context, one can consequently face, as for the case of (11), the sign decision problem due to the random phase of the effective charge.

Relation (11) is analog to the usual form of the dielectric response function with $\mathrm{N}$ optical modes [25]. For clarity, the small damping approximation is also assumed, in which case the TO frequencies are close to the poles of (11). The equivalence of (11) to its factored form, usually known as the Kurosawa form, is

$$
-n_{i}^{2} n_{j}^{2} \rho_{i j k}(\omega)=4 d_{k i j} \prod_{m}^{N} \frac{\omega_{L O, m}^{2}-\omega^{2}+i \gamma_{L O, m} \omega}{\omega_{T O, m}^{2}-\omega^{2}+i \gamma_{T O, m} \omega}
$$

It should be noted that (12) is valid for polar crystals that belong to orthorhombic and higher symmetry point groups. Based on the similarity form of (11) and the sum form of the dielectric response function, one can deduce the nonlinear analog of the oscillator strength, that is,

$$
B_{k i j, m}=\frac{a_{i j, m} Z_{k, m}}{\omega_{T O, m}^{2}}
$$

Note that $B_{k i j, m}$ is the transpose of a $3 \times 6$ third rank tensor and represents the individual lattice contribution to the E coefficient.

Relation (13) indicates that the square of the $B_{k i j, m}$ component can be related to the Raman cross-section and is therefore measurable from the Raman scattering experiments. Alternatively, one can have access to the Raman polarizability once $B_{k i j}$ is calculated. To do so, let us use the Kurosawa form of (12) to derive, as this is usually performed for the calculation of the effective charge parameter, the component of the $B_{k i j}$ tensor [25]:

$$
B_{k i j, q}=4 d_{k i j} \frac{\left(\omega_{L O, q}^{2}-\omega_{T O, q}^{2}-i \gamma_{L O, q} \omega_{T O, q}\right)}{\left|\omega_{T O, q}\right|^{2}} \frac{\prod_{q}\left(\omega_{L O, q}^{2}-\omega_{T O, q}^{2}-i \gamma_{L O, q} \omega_{T O, q}\right)}{\prod_{q \neq n}\left(\omega_{T O, q}^{2}-\omega_{T O, n}^{2}-i \gamma_{T O, n} \omega_{T O, n}\right)}
$$

Finally, the overall contribution of vibrational modes to the EO coefficient due to a given symmetry type is

$$
-n_{i}^{2} n_{j}^{2} r_{i j k}=4 d_{k i j}+\sum_{m}^{N} \operatorname{Re}\left(B_{k i j, m}\right)
$$


where $\mathrm{N}$ is the total number of polar vibrational modes per symmetry type and can be obtained by accounting for its order of degeneracy. For instance, in the symmetry point group of $\mathrm{LiNbO}_{3}, \mathrm{~N}=4 \times 1$ for the A1 symmetry type, while $\mathrm{N}=9 \times 2$ for the off-diagonal elements of the Raman tensor associated with the E normal modes.

As indicated above $\left(\rho_{i j k}(0)=r_{i j k}\right)$, it can easily be established by setting $\omega=0$ in (12):

$$
r_{i j k}=\frac{-4 d_{k i j}}{n_{i}^{2} n_{j}^{2}} \prod_{m=1}^{N} \frac{\omega_{L O, m}^{2}}{\omega_{T O, m}^{2}}
$$

Expression (16) can be considered as the nonlinear version of the Lyddane-SachsTeller (LST) relation because it connects the high frequency $d_{k i j}$ nonlinear dielectric tensor element to the linear clamped electro-optical coefficient. The main difference between (15) and (16) relies on the knowledge of the Raman linewidths. The Pockels EO coefficients could be determined using (16), even for overdamped Raman structures, provided that one use the statistical moments to calculate the statistical mean values of the TO and LO frequencies [25].

Furthermore, from the Miller index defined as $\chi_{i j k} /\left(n_{i}^{2}-1\right)\left(n_{j}^{2}-1\right)\left(n_{k}^{2}-1\right)$, one can deduce from (15) or (16) some relations which connect the electro-optical coefficient with other interesting nonlinear coefficients, such as the polarization optical coefficients or the Miller tensor components, that is,

$$
r_{i j k} \approx-2 n_{k}^{2} \Delta_{i j k} \prod_{m=1}^{N} \frac{\omega_{L O, m}^{2}}{\omega_{T O, m}^{2}}
$$

where $\Delta_{i j k}$ is the Miller index tensor component.

Now, if there are no external probes such as temperature, pressure, and others and considering that the refractive indices are not dependent on the exciting laser light (the change of the crystal bond length being negligible under the laser excitation), the relative error of the electro-optical coefficient due to the Raman scattering measurements can be calculated by logarithmically differentiating the LST relation (16), e.g.,

$$
\frac{\delta r_{i j k}}{r_{i j k}}=\frac{1}{\pi} \sum_{m}\left(\frac{\gamma_{T O, m}}{v_{T O, m}}-\frac{\gamma_{L O, m}}{v_{L O, m}}\right)
$$

with $v=\omega / 2 \pi$, where $v$ is the Raman wavenumber.

Roughly speaking, the Raman apparatus measures $v=\omega / 2 \pi$, which is then converted into wavenumbers; for that reason, Equation (18), written in terms of wavenumbers, is of practical interest for the calculation of the relative error. Relation (16) is very important because it points out that there is no need to extract the Raman polarizabilities from the complicated measurements of the absolute Raman intensity, which needs, additionally, the sign decision of the root square of the measured Raman scattering cross-section. The classical LST relation connects the high-frequency dielectric coefficient to the static dielectric constant through the lattice resonance frequencies and so on regarding the d-NLO and the EO coefficients (Relation 16). This means that the nonlinear optical constants of p order at zero and infinite energies are related to the fundamental vibrational energies. Since this is true for $p=1$ and $p=2$, it would be strongly motivating to verify semi-empirically the validity of such a relation for $p=3,4, \ldots$

\section{Results and Discussion}

First of all, we are only interested in the calculations of the linear clamped EO coefficients through (16), which requires the following data: the TO and LO fundamental wavenumbers associated with the selected Raman tensor element, the third-order nonlinear dielectric susceptibility components, and the diagonal element of the high-frequency linear dielectric tensor $\left(\epsilon_{0} n_{l l}^{2} ; 1=1,2,3\right.$; and $\epsilon_{0}$ is the vacuum permittivity). Unless otherwise specified, the data under consideration arise from the published experimental outputs. As stated 
in Section 1, the materials under study are uniaxial ferroelectric crystals. Some of them are negative uniaxial crystals (LN, BTO, PTO, and KNO) in which case the diagonal elements of the high frequency linear dielectric tensor are $\epsilon_{0} n_{e}^{2}=\epsilon_{0} n_{x}^{2}, \epsilon_{0} n_{y}^{2}=\epsilon_{0} n_{o}^{2}, \epsilon_{0} n_{o}^{2}=\epsilon_{0} n_{z}^{2}$ where $n_{0}$ and $n_{e}$ are the ordinary and extraordinary refractive indices, respectively, while LT is a positive uniaxial crystal with tensor diagonal element $\epsilon_{0} n_{x}^{2}=\epsilon_{0} n_{o}^{2}, \epsilon_{0} n_{y}^{2}=\epsilon_{0} n_{o}^{2}$, $\epsilon_{0} n_{z}^{2}=\epsilon_{0} n_{e}^{2}$. Accordingly, the dielectric axes of the d-NLO coefficients remain unaffected by these distinctions [26]. The DFT calculations are performed with the crystal atomic positions given in the crystallographic frame, which seems to coincide apparently with the negative uniaxial frame.

\subsection{EO Coefficients of the $L N$ and LT Crystals}

The LN crystal family has experienced renewed interest for several decades for fundamental studies and applications in optics and electronics because of its optimal EO and NLO properties, which are also demonstrated in LT crystals. However, despite their similar performances, there are some specific differences regarding their optical constants and spectroscopic characteristics. At room temperature, LN and LT crystals have a rhombohedral structure with $3 \mathrm{~m}$ and $\mathrm{m} 3$ point groups respectively. The number of modes per symmetry type at the center of the reciprocal lattice is given by $4 A_{1}(z)+9 E(x, y)+5 A_{2}$, where the $A_{2}$ normal modes are Raman and IR inactive, while both $A_{1}$ and $\mathrm{E}$ are polar modes with the corresponding Raman tensor:

$$
A_{1}(z)=\left(\begin{array}{ccc}
a & 0 & 0 \\
0 & a & 0 \\
0 & 0 & b
\end{array}\right) ; \quad E(-x)=\left(\begin{array}{ccc}
0 & c & d \\
c & 0 & 0 \\
d & 0 & 0
\end{array}\right) ; \quad E(y)=\left(\begin{array}{ccc}
c & 0 & 0 \\
0 & -c & d \\
0 & d & 0
\end{array}\right)
$$

where $a, b, c$, and $\mathrm{d}$ are the Raman polarizability tensor elements; the $z, x$, or $y$ inside the parentheses indicates the polar direction of the $A_{1}$-mode or the E-mode and is usually noted by the letter $\mathrm{k}$ in the indices of the $\mathrm{EO}$ tensor component, while the ij indices are usually contracted following the Voigt rule. It is important to note that the matrix element in (19), that is, the yy or 22 component of the Raman tensor $E(y)$, is negatively signed.

The Raman frequencies of the $\mathrm{A}_{1}$ and $\mathrm{E}$ symmetry types of the LN crystal considered here for calculation purposes are listed in Table 1 . For the $3 \mathrm{~m}$ symmetry point group, there are four independent nonlinear dielectric susceptibility components with values spread as follows: $d_{22}=2.1$ to $3.6 \mathrm{pm} / \mathrm{V} ; d_{31}=-4.7$ to $-6.8 \mathrm{pm} / \mathrm{V}$; and $d_{33}=-30.8$ to $-37.6 \mathrm{pm} / \mathrm{V}$ [27]. The well-known squared refractive indices of LN crystals are $n_{e}^{2}=4.6$ and $n_{o}^{2}=5$. Both experimental d-NLO coefficients and refractive indices of the LT congruent crystals under consideration are from [28]. The activated Raman E-modes of the LT crystal remain up to now the object of controversial reports, thus meaning that the Raman peaks corresponding to the E symmetry type have not yet been correctly assigned, despite several works [29-32] attempting to accomplish this. On the contrary, the $A_{1}$ activated optical phonons, being well assigned, will be taken into consideration (Table 1) to compute the $r_{13}$ and $r_{33}$ Pockels EO coefficients. The other EO coefficients could, of course, be calculated using (16) with the correctly assigned E-modes frequencies, if any. On the other hand, the optical constants considered for the calculations concern the congruent compound, in which case the connection of Raman to EO coefficients is shown to depend on the crystal composition [31].

Now, the EO coefficients can be calculated using (15), which needs, on the other hand, the linewidths of the Raman peaks. Unfortunately, most of the recently published Raman spectra of LN and LT crystals do not account for this type of spectroscopic data. For this reason, the nonlinear LST relation (16) will be systematically used instead (15). To calculate the $r_{i j k}$ coefficients, it is important to also account for the number of times the matrix element $\mathrm{ij}=\mathrm{ji}$ appears in the Raman tensor (19). For example, to calculate $r_{51}=r_{42}$, the xz or yz appears twice in the E(-x) or E(y) Raman tensors, contrary to the 22 matrix element that appears only once. The calculation of the $B_{22}$ or $r_{22}$ requires a little caution because of the sign of the $d_{22}$ component of the d-NLO tensor and the already mentioned sign 
of the 22 component of the Raman tensor $\mathrm{E}(\mathrm{y})$ in (19). The calculated values of the EO coefficients of the lithium niobate and of the lithium tantalate single crystals are shown in Table 1, together with others given for the purpose of comparison. In Table 1, satisfactory agreement with those published elsewhere can be observed.

Table 1. TO and LO Raman frequencies $\left(\mathrm{cm}^{-1}\right)$ and calculated Pockels EO coefficients (pm/V) due to the $\mathrm{A}_{1}$ and $\mathrm{E}$ normal modes and to the $\mathrm{A}_{1}$-modes in LN and LT crystals, respectively. Other EO coefficients are also displayed.

\begin{tabular}{|c|c|c|c|c|c|c|c|}
\hline \multicolumn{5}{|c|}{ LN } & \multicolumn{3}{|c|}{ LT } \\
\hline \multicolumn{3}{|c|}{$\mathrm{A}_{1}$-mode [33] } & \multicolumn{2}{|c|}{ E-mode [33] } & \multicolumn{3}{|c|}{$\mathrm{A}_{1}$-mode [29] } \\
\hline & $\mathrm{TO}$ & LO & TO & LO & & $\mathrm{TO}$ & LO \\
\hline & 253 & 271 & 152 & 198 & & 204 & 245 \\
\hline & 276 & 333 & 237 & 240 & & 252 & 347 \\
\hline & 335 & 431 & 262 & 297 & & 366 & 455 \\
\hline & 632 & 873 & 322 & 343 & & 597 & 866 \\
\hline & & & 366 & 425 & & & \\
\hline & & & 434 & 455 & & & \\
\hline & & & 579 & 660 & & & \\
\hline & & & 670 & 743 & & & \\
\hline & & & 735 & 878 & & & \\
\hline & $r_{13}$ & $r_{33}$ & $r_{22}$ & $r_{51}$ & & $r_{13}$ & $r_{33}$ \\
\hline Present & 6.85 & 28.96 & 4.63 & 18.88 & Present & 1.96 & 30.07 \\
\hline [34] & 7.7 & 28.7 & 3.4 & 18.2 & [28] & 4.6 & 30.5 \\
\hline [11] & 10.37 & 28.39 & 4.88 & 16.02 & [24] & 3.90 & 17.1 \\
\hline [24] & 2.58 & 3.903 & 1,30 & 6.26 & [35] & 8.4 & 30.5 \\
\hline
\end{tabular}

\subsection{EO Coefficients of Crystal of the Perovskite Structure: BTO, PTO, and KNO}

Barium titanate and potassium niobate are interesting substances because of the sequence of the temperature-dependent structural phase transitions that they undergo [36-38]. However, only one structural phase transition occurs at $766 \mathrm{~K}$ for PTO crystals [39]. The crystals of perovskite structure have recently been known for a revival of interest in a fundamental scheme for DFT studies of the ferroelectric-paraelectric phase transition $[40,41]$ and in an application scheme for their photovoltaic properties as detectors for visible light communication [42]. The computations of EO coefficients of these crystals are not considered by [13] in their study of the EO properties of ferroelectric materials using the BCM method, and in an attempt to fill this gap, [10,11] use the first-principles calculations within the DFT method to determine the EO coefficients of some crystals of the perovskite structure, thus attempting to confirm the success of their method applied to linear EO coefficients of the LN crystal. Our preliminary target is to validate our nonlinear LST relation from the comparison with the output of the ab initio DFT method and the experimental results which concern the aforementioned crystals. To this end, we based our computations on the crystal parameters that correspond to the tetragonal phase (4 $\mathrm{mm}$ point group) occurring at room temperature (BTO) and at high temperature (KNO), respectively. At the zero point of the reciprocal lattice, the number of the expected normal vibrations can be defined as follows: $3 A_{1}+4 E+3 B_{1}$. The $B_{1}$-irreducible representation is non-polar, while the $A_{1}$ and $E$ symmetry types are both Raman and IR active modes with the associated Raman tensor polarizabilities:

$$
A_{1}(z)=\left(\begin{array}{ccc}
a & 0 & 0 \\
0 & a & 0 \\
0 & 0 & b
\end{array}\right) \quad E(x)=\left(\begin{array}{lll}
0 & 0 & e \\
0 & 0 & 0 \\
e & 0 & 0
\end{array}\right) \quad E(y)=\left(\begin{array}{lll}
0 & 0 & 0 \\
0 & 0 & e \\
0 & e & 0
\end{array}\right)
$$

where the letter $x, y$, or $z$ and $a, b, c$, and $e$, corresponding to the vector and tensor components, respectively, have the same meaning as specified above. It appears from (11) and 
(20) that one can calculate only $r_{x x z}, r_{y y z}, r_{z z z}, r_{x z x}$, and $r_{y z y}$, which can be reduced to three independent coefficients: $r_{13}, r_{33}$, and $r_{51}$.

The parameters needed for the EO calculations of the aforementioned ferroelectric materials are listed in Table 2, in which the first set of data concerning the BTO crystals consists of the Raman frequencies calculated within the DFT method [43], the high frequency linear dielectric constants, and the d-NLO coefficients. The second set and the other following sets of data regarding the BTO, PTO, and $\mathrm{KNO}$ arise from published experimental results (Table 2).

Table 2. Raman frequencies $\left(\mathrm{cm}^{-1}\right)$ of BTO, PTO, and KNO; the corresponding refractive indices; and the d-NLO coefficients (pm/V), respectively. Some d-NLO coefficients of the KNO material [44,45] can also be considered for EO calculations if desired.

\begin{tabular}{|c|c|c|c|c|c|}
\hline & & BTO & BTO & PTO & KNO \\
\hline \multicolumn{2}{|c|}{ Normal mode } & [43] & [46] & {$[39,47]$} & [38] \\
\hline \multirow{6}{*}{$\mathrm{A}_{1}$} & TO1 & 161 & 178 & 140 & 190 \\
\hline & LO1 & 180 & 189 & 194 & 192 \\
\hline & TO2 & 302 & 276 & 359 & 295 \\
\hline & LO2 & 452 & 471 & 465 & 423 \\
\hline & TO3 & 507 & 515 & 647 & 600 \\
\hline & LO3 & 705 & 725 & 795 & 835 \\
\hline \multirow{16}{*}{ E } & TO1 & $161 i$ & 38 & 89 & 79 \\
\hline & LO1 & 162 & 180 & 130 & 191 \\
\hline & $\mathrm{TO} 2$ & 167 & $180 \mathrm{~h}$ & $220 \mathrm{~h}$ & $191 \mathrm{~h}$ \\
\hline & LO2 & 284 & 308 & 290 & 199 \\
\hline & TO3 & 284 & 308 & 290 & 199 \\
\hline & LO3 & 444 & 466 & 440 & 418 \\
\hline & TO4 & 457 & 498 & 508 & 521 \\
\hline & LO4 & 641 & 722 & 720 & 822 \\
\hline & \multirow{3}{*}{$\begin{array}{l}\mathrm{n}_{\mathrm{x}}^{2} \\
\mathrm{n}_{\mathrm{z}}^{2}\end{array}$} & [43] & [48] & [49] & [50] \\
\hline & & 6.48 & 5.19 & 6.64 & 5.55 \\
\hline & & 5.84 & 5.05 & 6.63 & 5.03 \\
\hline & \multirow{5}{*}{$\begin{array}{l}d_{31} \\
d_{33} \\
d_{42} \\
d_{51}\end{array}$} & [43] & [27] & [51] & [52] \\
\hline & & -11.09 & -15.70 & -42.80 & -15.8 \\
\hline & & -18.31 & -6.8 & +8.5 & -27.4 \\
\hline & & & & & -18.30 \\
\hline & & -11.09 & -17 & -37.9 & -16.5 \\
\hline
\end{tabular}

The first column of Table 2 shows the lowest frequency E-mode which is imaginary, i.e., 161i [43]. However, based on the first-principles calculations, [53] found this mode at $178 \mathrm{i}$, which is very close to the Raman structure observed [46] at $180 \mathrm{~cm}^{-1}$ that we labeled by the letter $\mathrm{h}$. Similar to the measured Raman frequencies recorded on BTO, the soft mode in the Raman spectra of PTO and KNO is also distinguished by the same label. It is important to note that the lowest frequency modes, that is, the Raman peaks activated at 38 $\mathrm{cm}^{-1}$ for BTO, at $89 \mathrm{~cm}^{-1}$ for PTO, and at $79 \mathrm{~cm}^{-1}$ for $\mathrm{KNO}$, are not the soft mode, in order to avoid confusion with [53]. It can be remarked from Table 2 that we have reassigned the vibrational frequencies of the E symmetry type of $\mathrm{KNO}$ by using parallel mode assignments performed on the BTO Raman spectrum [46] and by taking into account the list of the theoretical frequencies sorted out by [53], who rejected the $280 \mathrm{~cm}^{-1}$ vibrational frequency present in the IR reflection spectra of KNO [38]. The Raman frequencies of PTO crystals recorded by $[39,47]$ present equal values, except for their assigned $\mathrm{E}(\mathrm{LO} 4)$, and were also reassigned following the same procedure. We considered the h-labeled frequency as a guide to perform E-mode frequency assignments to avoid the inversion of the TO-LO couples, thus leading to a negative dielectric constant through the classical LST relation. 
The calculated EO coefficients of the BTO, PTO, and KNO crystals by using (16) are displayed in Table 3 together with those published elsewhere (see references therein) and are in fairly good agreement. On the other hand, one can also observe a satisfactory agreement between the results with * and those of [24].

Table 3. EO coefficients (pm/V) of BTO, PTO, and KNO calculated using (16). Present and other EO coefficients determined elsewhere: see the associated references.

\begin{tabular}{lccccc}
\hline & & $r_{13}$ & $r_{33}$ & $r_{42}$ & $r_{51}$ \\
\hline $\mathrm{BaTiO}_{3}$ & Present & 15.17 & 6.56 & & 1640.21 \\
& Present & 5.71 & 11.62 & & \\
& Exp [54] & 10.2 & 40 & & 1650 \\
& Exp [55] & & & & \\
& Cal [24] & 5.51 & 11.6 & & 118.07 \\
& Cal [11] & 12.68 & 30.84 & & 30.53 \\
\hline $\mathrm{PbTiO}_{3}$ & Present & 10.78 & -3.32 & & 350.01 \\
& Exp [27] & 13.8 & 5.9 & & 105 \\
& Cal [11] & 8.98 & 5.88 & & 27.8 \\
$\mathrm{KNbO}_{3}$ & Present & 8.41 & 17.37 & 362.73 & \\
& Exp [56] & 28 & 64 & 380 & 12.108 \\
& Exp [57] & 10 & 34 & 360 & \\
& Cal [24] & 4.26 & 17.055 & 12.108 & 350.3 \\
& Cal [15] & & & & \\
& & & & & \\
&
\end{tabular}

* calculated EO coefficients from data of [43].

\subsection{Discussion}

We tested our nonlinear LST relation by calculating the clamped linear EO coefficients of some ferroelectric oxides of the $\mathrm{ABO}_{3}$ chemical species such as crystals of the LN structure (LN and LT) and those of perovskite structure (BTO, PTO, and KNO). The obtained results compared to the directly measured ones and other EO coefficients calculated from DFT or BCM methods are in good agreement. Further analysis of Table 3 shows that relation (16) intriguingly leads to results very close to the output of DFT calculations performed using LDA $[10,11,24]$. This is illustrated by the present results labeled by an asterisk. Some disagreements concerning mainly the $r_{33}$ coefficient of BTO and KNO can be explained by the lack of correction due to the mode damping coefficients or the fitted values of the Raman linewidths, which are often sensitive to temperature effect. Since the tetragonal phase of KNO takes place at high temperatures (585 C) [39], the corresponding vibrational spectra might be accompanied by broadening lines that impact the calculation of $r_{i j k}$ through (15) or, alternatively, increase the relative error $\delta r_{i j k} / r_{i j k}$ (18). Based on the fitted values of $\gamma_{T O}, \gamma_{L O}, \omega_{T O}$, and $\omega_{L O}$ extracted from the Raman spectrum of the $A_{1}$ normal modes [38], we estimated $\delta r_{13} / r_{13}=\delta r_{33} / r_{33}=0,5$, thus correcting $r_{13}$ to $12 \mathrm{pm} / \mathrm{V}$ and $r_{33}$ to $25 \mathrm{pm} / \mathrm{V}$, close to the experimental results (Table 3). Unfortunately, we have no equivalent fitted spectral parameters for the BTO crystal. Nevertheless, a comparison of the experimental Raman spectrum and that calculated from first-principles within DFT (see [43], page 9), clearly shows that neglecting the damping coefficients could result in erroneously calculated EO coefficients of BTO, even by accounting for the scissors shifts corrections acting on the values of the DFT-based calculations of the d-NLO coefficients or the refractive indices [58]. The PTO crystal presents a very low spontaneous birefringence that can prevent direct measurement of the $r_{51}$ coefficient. Since its $d_{33}$ coefficient is positive [51], we obtained from (16) negative values of the $r_{33}$ coefficient in conflict with the experimental result. On the contrary, our calculated $r_{13}$ agrees very well with that calculated $[11,54]$ and is slightly different from the experimental value because at room temperature and far from the phase transition point, the linewidths of the Raman peaks contribute to a lesser extent $[39,43]$. As mentioned above, there is not, to our knowledge, 
a measured $r_{51}$ value for the PTO crystal, thus leaving open the theoretical determination of this coefficient, which is found in this work to be four times greater than other calculated ones $[10,11,24]$. However, if the contribution of the lowest frequency mode is rejected, it results from (16) that $r_{51}=32.76 \mathrm{pm} / \mathrm{V}$, which is nearly of the same order of magnitude as those theoretically calculated according to the LDA. As a consequence, our $r_{51}$ Pockels coefficient of the PTO compound is more reliable than others computed from the DFT-based method.

The high value of the $r_{51}$ coefficient of the BTO crystal, assumed to originate from the relaxation process, is strongly enhanced at the $\mathrm{GHz}$ frequency scale [59]. However, since the soft mode frequency is imaginary, as suggested by the first-principles studies [53], it may consequently result in an excess of the effective charge parameter, thus creating an apparent inversion of the LO-TO mode assignments of frequencies limited by the soft mode and the hardest mode [60]. This fact, also observed in the IR reflection spectra [36] of the $\mathrm{CaTiO}_{3}$ crystal, seems to be a typical behavior of the $\mathrm{ABO}_{3}$ oxide perovskite crystals. We have reassigned the published E-mode frequencies of KNO and PTO crystals using parallel mode assignments performed on BTO crystals [46] based on the imaginary frequency peak position (Table 3). The anomalous frequency of the soft mode, which is responsible for the excess of the effective charge, also affects the value of the $B_{k i j}$ coefficient through (14). This situation, in conjunction with the lowest frequency mode position (typically less than $100 \mathrm{~cm}^{-1}$ ), can finally be considered as the main physical cause of the outstanding values of the $r_{51}$ coefficient of the $\mathrm{ABO}_{3}$ perovskite crystals. This scenario is not encountered while calculating the EO coefficients of the LN crystal.

\section{Conclusions}

We derived the factored form of the nonlinear analog of the dielectric response function, from which we established two simple formulas that connect the vibrational spectroscopic data, the high frequency nonlinear dielectric coefficients, with the EO coefficient. The nonlinear LST relation (16), being one of these formulas, is systematically used for the computations of the EO coefficients of ferroelectric materials of the $\mathrm{ABO}_{3}$ crystal family. Our approach is also able to estimate the relative errors due to the measurements of the optical phonon linewidths and the positions of the corresponding peak from the vibrational spectra. The obtained results show an overall agreement with both experimental and other calculated EO coefficients from DFT and BCM methods, and this agreement additionally points out an alternate way dedicated to the computations of EO coefficients from the computed Raman frequencies on the basis of first-principles calculations. The derived nonlinear LST relation, together with the classical LST relation, can efficiently help to assess the Raman mode frequency assignments of ferroelectric crystals. We also calculated the $r_{51}$ or $r_{42}$ coefficients, which have not been correctly calculated until now, and found that the lowest frequency mode certainly originated from the relaxation process, and the anomalous behavior of the soft mode is definitively the major cause of the outstanding values of the coefficients of uniaxial oxide perovskite crystals. It should be more interesting to study the performances of the present model by extending the computations of the EO coefficients to overdamped Raman profiles associated with materials such as ceramic PLZT and to crystals with low symmetry such as biaxial $\mathrm{KNbO}_{3}$ at room temperature or $\mathrm{KTiOPO}_{4}$ at room and high temperatures.

Author Contributions: Conceptualization, B.M.; methodology, B.M.; software, B.M.; validation, E.B., P.B. and B.M.; formal analysis, P.B.; investigation, B.M.; resources, E.B.; data curation, E.B.; writing-original draft preparation, B.M.; writing—review and editing, B.M.; visualization, E.B.; supervision, P.B.; project administration, P.B.; funding acquisition, E.B. All authors have read and agreed to the published version of the manuscript.

Funding: This research received no external funding.

Data Availability Statement: Not applicable. 
Acknowledgments: The authors would like to thank Germano Montemezzani for the critical reading of the manuscript.

Conflicts of Interest: The authors declare no conflict of interest.

\section{References}

1. Poulet, H. Proprits pizo-optiques etlectro-optiques de la blende. J. Phys. Radium 1955, 16, 237-238. http://doi.org/10.1051/ jphysrad:01955001603023701. [CrossRef]

2. Loudon, R. The Raman effect in crystals. Adv. Phys. 1964, 13, 423 [CrossRef]

3. Mirochnichenko, G.P.; Kislev, A.D.; Trifanov, A.I.; Gleim, A.V. Algebraic approach to electro-optic modulation of light: Exactly solvable multimode quantum model. JOSA B 2017, 34, 1177. doi: 10.1364/JOSAB.34.001177. [CrossRef]

4. Liu, J.-M. Photonics Devices; Oxford University Press: New York, NY, USA, 2005.

5. Tshang, M.; Shapiro, J.H.; Lloyd, S. Quantum theory of optical temporal phase and instantaneous frequency. Phys. Rev. A 2008, 78, 053820. [CrossRef]

6. Tshang, M.; Shapiro, J.H.; Lloyd, S. Quantum theory of optical temporal phase and instantaneous frequency. II. Continuous-time limit and state-variable approach to phase-locked loop design. Phys. Rev. A 2009, 79, 053843. [CrossRef]

7. Kaminow, I.P.; Johnston, W.D., Jr. Quantitative Determination of Sources of the Electro-Optic Effect of $\mathrm{LiNbO}_{3}$ and $\mathrm{LiTaO}_{3}$. Phys. Rev. 1967, 160, 519. [CrossRef]

8. Johnston, W.D., Jr. Nonlinear Optical Coefficients and the Raman Scattering Efficiency of LO and TO Phonons in Acentric Insulating Crystals. Phys. Rev. B 1970, 1, 3494. [CrossRef]

9. Knoll, P.; Kuzmany, H. Nonlinear-optical properties and signs of the Raman tensor for LiGaO 2. Phys. Rev. B 1984, $29,2221$. [CrossRef]

10. Veithen, M.; Gonze, X.; Ghosez, P. First-Principles Study of the Electro-Optic Effect in Ferroelectric Oxides. Phys. Rev. Lett. 2004, 93, 187401. [CrossRef] [PubMed]

11. Veithen, M.; Gonse, X.; Ghosez, P. Nonlinear optical susceptibilities, Raman efficiencies, and electro-optic tensors from firstprinciples density functional perturbation theory. Phys. Rev. B 2005, 71, 125107. [CrossRef]

12. Levine, B.F. Bond-Charge Calculation of Nonlinear Optical Susceptibilities for Various Crystal Structures. Phys. Rev. B 1973, 7, 2600. [CrossRef]

13. Shih, C.-C.; Yariv, A. A theoretical model of the linear electro-optic effect. J. Phys. C Solid State Phys. 1982, 15, 825. [CrossRef]

14. Sastry, P.U. Linear electro-optical properties of tetragonal $\mathrm{BaTiO}_{3}$. Solid State Commun. 2002, 122, 41. [CrossRef]

15. Sastry, P.U. Electro-optical properties of tetragonal $\mathrm{KNbO}_{3}$. Pramana 2007, 68, 1001-1006. [CrossRef]

16. Lines, M.E. Bond-orbital theory of linear and nonlinear electronic response in ionic crystals. II. Nonlinear response. Phys. Rev. $B$ 1990, 41, 2383. [CrossRef]

17. Wang, F. Calculation of the electro-optical and nonlinear optical coefficients of ferroelectric materials from their linear properties. Phys. Rev. B 1999, 59, 9733. [CrossRef]

18. Chen, C.; Yang, H.; Wang, Z.; Liu, Z. A theoretical model to calculate linear electro-optic effect in crystals. Chem. Phys. Lett. 2004, 11, 222. [CrossRef]

19. Jazbinsek, M.; Zgonik, M. Material tensor parameters of LiNbO3 relevant for electro- and elasto-optics. Appl. Phys. B 2002, 74, 497. doi: 10.1007/s003400200818. [CrossRef]

20. Kaminow, I.P. Ferroelectricity; Weller, E.F., Ed.; Elsevier Publishing Co.: Amsterdam, The Netherlands, 1967.

21. Kaminow, I.P.; Turner, E.H. Temperature Dependence of Raman Scattering and the Electro-Optic Properties of CuCl. Phys. Rev. B 1972, 5, 1564. [CrossRef]

22. Born, M.; Huang, K. Dynamical Theory of Crystals Lattice; Clarendon: Oxford, UK, 1954.

23. Barker, A.S.; Loudon, R. Response Functions in the Theory of Raman Scattering by Vibrational and Polariton Modes in Dielectric Crystals. Rev. Mod. Phys. 1972, 44, 18. [CrossRef]

24. Cabuk, S. The nonlinear optical susceptibility and electro-optic tensor of ferroelectrics: First-principle study. Cent. Eur. J. Phys. 2012, 10, 239. doi: 10.2478/s11534-011-0079-3. [CrossRef]

25. Barker, A.S., Jr. Long-wavelength soft-modes, central peaks, and the Lyddane-Sachs-Teller relation. Phys. Rev. B 1975, $12,4071$. [CrossRef]

26. Roberts, D.A. Simplified Characterization of Uniaxial and Biaxial Nonlinear Optical Crystals: A Plea for Standardization of Nomenclature and Conventions. IEEE J. Quantum Electron. 1992, 28, 20057. [CrossRef]

27. Weber, M.J. (Ed.) Handbook of Laser Sciences and Technology. In Optical Materials; CRC Press: Boca Raton, FL, USA, 2003.

28. Casson, J.L.; Gahagan, K.T.; Scrymgeour, D.A.; Jain, R.K.; Robins, J.M.; Gopalan, V.; Sanders, R.K. Electro-optic coefficients of lithium tantalate at near-infrared wavelengths. J. Opt. Soc. Am. B 2004, 21, 1948-1952. [CrossRef]

29. Friedrich, M.; Schindlmayr, A.; Schmidt, W.G.; Sanna, S. LiTaO3 phonon dispersion and ferroelectric transition calculated from first principles. Phys. Status Solidi B 2016, 253, 683. doi: 10.1002/pssb.201552576. [CrossRef]

30. Margueron, S.; Bartasyte, A.; Glazer, A.M.; Simon, E.; Hlinka, J.; Gregora, I.; Gleize, J. Resolved E-symmetry zone-centre phonons in LiTaO3 and LiNbO3. J. Appl. Phys. 2014, 111, 104105. doi: 10.1063/1.4716001. [CrossRef] 
31. Kotristski, S.; Bourson, P.; Aillerie, M.; Fontana, M.D.; Kip, D. Quantitative evaluation of the electro-optic effect and second-order optical nonlinearity of lithium tantalate crystals of different compo-sitions using Raman and infrared spectroscopy. Appl. Phys. B 2006, 82, 423. doi: 10.1007/s00340-005-2046-4. [CrossRef]

32. Shi, L.; Kong, Y.; Yan, W.; Liu, H.; Li, X.; Xie, X.; Zhao, D.; Sun, J.; Chen, S.; Zhang, L.; et al. The composition dependence and new assignment of the Raman spectrum in lithium tantalate. Solid State Commun. 2005, 135, 251. doi: 10.1016/j.ssc.2005.04.024. [CrossRef]

33. Maimounatou, B.; Mohamadou, B.; Erasmus, R. Experimental and theoretical directional dependence of optical polar phonons in the LiNbO3 single crystal: New and complete assignment of the normal mode frequencies. Phys. Status Solidi B 2015, $253,573$. [CrossRef]

34. Kaminow, I.P.; Turner, E.H.; Barns, R.L.; Bernstein, J.L. Crystallographic and electro-optic properties of cleaved LiNb0 3. J. App. Phys. 1980, 51, 4379. [CrossRef]

35. Boyd, R.W. Nonlinear Optics; Academic Press Inc.: San Diego, CA, USA, 1992.

36. Kommandin, C.A.; Volkov, A.A.; Porodnikov, O.E.;Spektor, I.E.; Chuchupal, S.V. On the Problem of the LO-TO Splitting of the Soft Mode in $\mathrm{CaTiO}_{3}$. Phys. Solid State 2013, 55, 1896. doi: 10.1134/S1063783413060188. [CrossRef]

37. Nakamura, T. Soft phonon in $\mathrm{BaTiO}_{3}$. Ferroelectrics 1992, 137, 65. [CrossRef]

38. Fontana, M.D.; Metrat, G.; Servoin, J.L.; Gervais, F. Infrared spectroscopy in $\mathrm{KNbO}_{3}$ through the successive ferroelectric phase transitions. J. Phys. C 1984, 17, 483. [CrossRef]

39. Fontana, M.D.; Idrissi, H.; Kugel, G.E.; Wojcik, E. Raman spectrum in $\mathrm{PbTiO}_{3}$ re-examined: Dynamics of the soft phonon and the central peak. J. Phys. Condens. Matter 1991, 3, 8695. [CrossRef]

40. Veithen, M.; Ghosez, P. First-principles study of the dielectric and dynamical properties of lithium niobate. Phys. Rev. B 2002, 65, 214302. [CrossRef]

41. Paillard, C.; Torum, E.; Wirtz, L.; Bellaiche, L. Strain engineering of electro-optic constants in ferroelectric materials. Phys. Rev. Lett. 2019, 123, 087601. [CrossRef] [PubMed]

42. Mica, N.A.; Bian, H.; Manousiadis, P.; Jagadamma, L.; Tavakkolnia IHaas, H.; Tumbull, G.; Samuel, I. Triple-cation perovskite solar cells for visible light communications. Photonics Res. 2020, 8, A16. [CrossRef]

43. Hermet, P.; Veithen, M.; Ghosez, P. Raman scattering intensities in $\mathrm{BaTiO}_{3}$ and $\mathrm{PbTiO}_{3}$ prototypical ferroelectrics from density functional theory. J. Phys. Condens. Matter 2009, 21, 215901. doi: 10.1088/0953-8984/21/21/215901. [CrossRef]

44. Xue, D.; Zhang, S. Linear and nonlinear optical properties of $\mathrm{KNbO}_{3}$. Chem. Phys. Lett. 1998, 291, 401. [CrossRef]

45. Hewat, A.W. Cubic-tetragonal-orthorhombic-rhombohedral ferroelectric transitions in perovskite potassium niobate: Neutron powder profile refinement of the structures. J. Phys. C 1973, 6, 2559. [CrossRef]

46. Scalabrin, A.; Chaves, A.S.; Shim, D.S.; Porto, S.P.S. Temperature dependence of the A1 and E optical phonons in BaTiO 3 . Phys. Staus Solidi B 1977, 79, 731. [CrossRef]

47. Foster, C.M.; Li, Z.; Grimsditch, M.; Chan, S.-K.; Lam, D.J. Anharmonicity of the lowest-frequency A1(TO) phonon in PbTiO 3 . Phys. Rev. B 1993, 48, 10160. [CrossRef] [PubMed]

48. Shumate, M.S. Interferometric Measurement of Large Indices of Refraction. Appl. Opt. 1966 , 5, 327. [CrossRef] [PubMed]

49. Miller, R.C.; Northl, W.A. Further Measurements of Absolute Signs of Second-Harmonic-Generation Coefficients of Piezoelectric Crystals. Phys. Rev. B 1972, 5, 4931. [CrossRef]

50. Wiesendanger, E. Optical Properties of $\mathrm{KNbO}_{3}$. Ferroelectrics 1970, 1, 141. [CrossRef]

51. Sing, S.; Remeika, J.P.; Potopowicz, J.R. Nonlinear Optical Properties of Ferroelectric Lead Titanate. Appl. Phys. Lett. 1972, $20,135$. [CrossRef]

52. Xing, W.; Looser, H.; Wüest, H.; Arend, H. Progress in $\mathrm{KNbO}_{3}$ crystal growth. J. Cryst. Growth 1986, 78, 431. [CrossRef]

53. Zhong, W. King-Smith and Vanderbilt D Giant TO-LO Splittings in Perovskites Ferroelectrics. Phys. Rev. Lett. 1994, 72,3618 . [CrossRef]

54. Zgonik, M.; Bernasconi, P.; Duelli, M.; Schlesser, R.; Gunter, P.; Garret, M.H.; Rytz, D.; Zhu, Y.; Wu, X. Dielectric, elastic, piezoelectric, electro-optic, and elasto-optic tensors of BaTiO3 crystals. Phys. Rev. B 1994, 50, 5991. [CrossRef] [PubMed]

55. Jonston, A.R.; Weingart, J.M. Determination of the low-frequency linear electro-optic effect in tetragonal BaTiO 3 . J. Opt. Soc. Am. 1965, 55, 828-834. [CrossRef]

56. Gunter, P. Electro-Optics/Laser; Jerrard, H.G., Ed.; IPC Science and Technology Press: Guilford, UK, 1976.

57. Zgonik, M.; Schlesser, R.; Biaggio, I.; Voit, E.; Tscherry, J.; Gunter, P. Materials constants of $\mathrm{KNbO}_{3}$ relevant for electro- and acousto-optics. J. Appl. Phys. 1993, 74, 1287. [CrossRef]

58. Schmidt, F.; Riefer, A.; Schmidt, W.G.; Schindlmayr, A.; Imlau, M.; Sanna, S. Quasiparticle and excitonic effects in the optical response of $\mathrm{KNbO}_{3}$. Phys. Rev. Mater. 2019, 3, 054401. doi: 10.1103/PhysRevMaterials.3.054401. [CrossRef]

59. Fontana, M.D.; Laabidi, K.; Jeannot, B.; Maglione, M.; Julien, P. Relationship between electro-optic, vibrational and dielectric properties in $\mathrm{BaTiO}_{3}$. Solid State Commun. 1994, 92, 827. [CrossRef]

60. Gervais, F. Infrared dispersion in several polar-mode crystals. Opt. Commun. 1977, 22, 116. [CrossRef] 